Pensamiento Crítico Vol. 20 № 2, pp. 37-52

\title{
La importancia de la Alianza del Pacífico
}

The importance of the Pacific Alliance

[Recepción: Setiembre de 2015 / Conformidad: Octubre de 2015]

Pedro Barrientos Felipa ${ }^{1}$

\section{RESUMEN}

La Alianza del Pacífico es una nueva iniciativa de integración en Latinoamérica. Es parte del proceso que impulsa la globalización en la búsqueda de un mejor aprovechamiento de las oportunidades que se presentan en el mercado internacional. Oportunidades que se dan a través de nuevos procesos de producción, lo que representa un desafío: pasar de un país primario exportador a un país exportador con productos de mayor valor agregado. Sacar provecho de lo que se tiene y aprender para seguir creciendo. El artículo busca exponer el compromiso que tienen los países integrantes para avanzar hacia una nueva etapa que debe ser aprovechada para el beneficio equitativo de sus agentes económicos.

Palabras clave: Globalización, Alianza del Pacífico, acuerdos comerciales, integración latinoamericana.

1 Economista por la Universidad Ricardo Palma, Lima - Perú. Magíster en Administración con Concentración en Marketing por la Universidad del Pacífico, Lima - Perú. Doctor en Administración de Negocios Globales por la Universidad Ricardo Palma. Profesor principal de la Facultad de Ciencias Económicas, director de la Escuela de Economía Internacional; miembro titular del Instituto de Investigaciones Económicas de la Universidad Nacional Mayor de San Marcos. Correo electrónico: pbarrientosf@unmsm.edu.pe. 


\title{
Pensamiento Crítico Vol. 20. No 2
}

\begin{abstract}
The Pacific Alliance is a new initiative for integration in Latin America. It is part of the process that drives globalization in search of a better use of the opportunities presented in the international market. Opportunities provided by new production processes, which represents a challenge: moving from a primary exporting country to an exporting country with higher value added. Take advantage of what you have and learn to keep growing. This article explains the commitment that the countries to advance towards a new stage and should be harnessed for the equal benefit of their economic operators.
\end{abstract}

Keywords: Globalization, Pacific Alliance, trade agreements, latin american integration.

\section{Perú y la globalización}

El Perú tiene los suficientes recursos naturales que gozan de muchas probabilidades de desarrollarse en el mercado global, pero debe darle mayor valor agregado y, en este caso, se considera a los productos denominados "no tradicionales". Las barreras comerciales se han bajado en el país; sin embargo, hay otras barreras no comerciales que el mismo país se ha puesto interiormente y todavía no bajan a la velocidad que la globalización requiere. Se continúa en la etapa de productor de insumos, de commodities. Hay empresas peruanas que actúan globalmente como es el caso del Grupo Gloria $^{2}$, lo que demuestra la capacidad de las empresas peruanas para ingresar invirtiendo directamente en mercados extranjeros, lo cual debe ser el horizonte de toda empresa peruana, sea pequeña, mediana o grande, participando en el comercio internacional directa o indirectamente.

"Tiene que preguntarse: '¿Hasta qué punto mi país está avanzando o está quedándose atrás por efecto del aplanamiento del mundo? ¿Hasta qué punto se está adaptando y sacando partido de todas estas nuevas plataformas de colaboración y competición?' Como dijo, alardeando, aquel funcionario del banco chino a mi colega mexicano: China es el lobo. De todos los aplanadores, la entrada

2 El Grupo Gloria es un conglomerado industrial de capitales peruanos con negocios presentes en Perú, como también en Bolivia, Colombia, Ecuador, Argentina y Puerto Rico. Sus actividades se desarrollan en los sectores de lácteos y alimentos, en cemento, papeles, agroindustria, transporte y servicios. Publicado en www.grupogloria.com. Consultado el 25 de julio del 2014. 


\section{Pedro Barrientos Felipa}

de China en el mercado mundial es el más importante para los países en vías de desarrollo y para muchos de los desarrollados". Friedman (2006, p. 328).

La formación de bloques comerciales en el proceso de globalización favorece a un país, si las condiciones están dadas para el desarrollo empresarial. El Estado se convierte en un agente impulsor de las actividades de las empresas e inversionistas privados; depende de ellos si aceptan el desafío y estar preparados para enfrentar la competitividad internacional. La consolidación del bloque comercial es un proceso largo, al igual de los países integrantes en cuanto a ponerse entre ellos a la par en los indicadores estructurales de la sociedad, como la educación (se va a necesitar gente mucho mejor capacitada), aspectos sanitarios, de justicia, de seguridad, entre otros. Son estos los que le permiten tener un efecto de sinergia.

"La globalización de las economías ha generado una suma de problemas y oportunidades a las empresas y a los individuos que las dirigen. Quien llega tarde a entender su encantamiento, tendrá mayores problemas y menos oportunidades. Mayores problemas porque tendrán una mayor exigencia para cumplir los estándares que han establecido quienes llegaron con anterioridad, y menos oportunidades porque lo que se deja pasar alguien lo toma, y volver a querer recuperarlo se hace más complicado. Si la empresa está en capacidad de aprovechar una oportunidad, debe aprovecharla; la oportunidad se representa en consecuencias financieras que los inversionistas deben saber apreciar y exigir a quien dirige la empresa y administra los productos". Barrientos (2009, p. 62)

Perú es un país que está siendo observado como uno de los que mejor comportamiento macroeconómico tiene entre los diversos países que quieren una vida mejor para sus ciudadanos. Jaramillo y Silva-Jáuregui (2011) mencionan la importancia de determinados factores económicos y políticos que permiten consolidar el proyecto de crecimiento. Aunque lo que citan inicialmente en un comentario amplio, es decir, no llega al detalle, es válido el pronunciamiento que sirve como una fuente para elaboración de guías y así estar en posibilidades de adaptarse a las oportunidades de la globalización. La globalización fuerza a la integración de los países, más aún si se toma lo expuesto por Vásquez Barquero (2005), en cuanto a que la globalización está dando origen "a un nuevo orden internacional y a una nueva división internacional del trabajo", lo que coincide con lo que menciona Ohmae (2008). Las decisiones comerciales 


\section{Pensamiento Crítico Vol. 20. No 2}

están influidas por este nuevo concepto. La integración que realiza Perú, además de las razones citadas, es conveniente que se realice porque este proceso se está dando en todo el mundo. La integración económica se ha acelerado, así como la intensidad de competencia entre países y empresas.

Los socios de la Alianza del Pacífico tienen varios tratados comerciales comunes, lo que explica su experiencia en este tipo de relación. De ahí que puede plantearse cómo identificar un beneficio específico que origina la Alianza considerando que ya existen acuerdos entre los miembros y con otros países que son parte del mercado objetivo que se plantean los cuatro países. Burgos et al. (2003, p. 20) mencionan que la globalización trae consigo cambios profundos en el espacio y el tiempo. La reestructuración del espacio creado por el proceso de globalización ha variado.

También es importante la idea de Ohmae (2008, p.8) en cuanto a que "el escenario global exige un nuevo guion. A su vez, este exige que los actores principales (tanto individuos como instituciones, ya sean corporaciones, sindicatos, grupos de influencia, inversionistas, gobiernos regionales y nacionales) cambien su manera de actuar y pensar". La realidad frente a la globalización ha cambiado y no es posible salirse del trazo que ha definido. Las respuestas tienen que ser prontas a la circunstancia y tener un carácter flexible para acomodarse a la nueva economía, siempre considerando un pensamiento nacional de largo plazo, en el que los principales agentes económicos y sociales se comprometen.

Pero, también es necesario tener presente lo expuesto por Rodrik (2011, p. 202) en cuanto a que "el crecimiento y el desarrollo económicos son posibles solamente a través de la acumulación de capacidades a lo largo del tiempo, en áreas que van desde la educación y las tecnologías hasta las instituciones públicas. La globalización no genera esas capacidades por sí misma; simplemente permite que los países saquen mejor partido de las capacidades que ya se poseen". Entonces, los beneficios de la globalización no vienen al interior de las fronteras nacionales, sino que los países tienen que ir a buscarlos pues están esperando que algún país, representado a través de sus empresas, los haga suyos y a su vez los comparta. 


\section{Pedro Barrientos Felipa}

\section{2. ¿Qué es la Alianza del Pacífico?}

La Alianza del Pacífico se define a sí misma como una iniciativa de integración regional creada el 28 de abril de 2011 por Chile, Colombia, México y Perú. Esta tiene por objetivos:

- Construir, de manera participativa y consensuada, un área de integración profunda para avanzar progresivamente hacia la libre circulación de bienes, servicios, capitales y personas.

- Impulsar un mayor crecimiento, desarrollo y competitividad de las economías de las partes, con miras a lograr mayor bienestar, superar la desigualdad socioeconómica e impulsar la inclusión social de sus habitantes.

- Convertirse en una plataforma de articulación política, integración económica y comercial, y proyección al mundo, con énfasis en la región Asia-Pacífico.

Acuña (2013) expone que la base para la conformación de la Alianza del Pacífico son los acuerdos comerciales previamente firmados entre sus miembros, además de las obligaciones asumidas dentro de los foros multilaterales y regionales de los que hacen parte. Los cuatro países fundadores son miembros de la Organización Mundial del Comercio (OMC) y de la Asociación Latinoamericana de Integración (ALADI) y Colombia y Perú hacen parte de la Comunidad Andina (CAN).

"La Alianza del Pacífico representa un mercado de 200 millones de consumidores con ingresos per cápita promedio de U $\$ 13.500$, que representan el 34,8\% del PIB de Latinoamérica, exporta US $\$ 440$ mil millones y representan el $50 \%$ de las importaciones de la región, lo que lo hace atractivo para convertirse en una plataforma de articulación política, de integración económica y comercial, y de proyección al mundo, con especial énfasis al Asia Pacífico" (San Francisco, 2013, p. 5).

Serrano (2012) plantea varias ideas que deben considerarse en el análisis de la Alianza. Así, sostiene que en Iberoamérica hay tantas organizaciones internacionales que terminan confundiendo sus competencias con otras, lo que las hace perder su eficacia y solo se limitan a sobrevivir. Asimismo, cuestiona la no inclusión de Estados Unidos, que tiene amplia experiencia en el territorio asiático, o es que fue simplemente una for-

ma de disminuir la influencia de este país. China puede estar creando un espejismo que 


\section{Pensamiento Crítico Vol. 20. N²}

puede llevar a los países del bloque a un túnel sin salida, política y económicamente. Entonces, por qué no acercarse más a Australia, Japón, Indonesia, Singapur. Son temas que los países deben resolver y que hay que observar con atención y esperanza.

\section{Como menciona Ronderos ${ }^{3}$ :}

"La Alianza sigue siendo una expectativa porque todavía ni el marco, ni el protocolo comercial están aprobados. Lo que realmente rige en este momento a la AP son los acuerdos de libre comercio bilaterales que ya existen entre México y Chile, Chile y Perú, Perú y Colombia, etc.,... Eso es un plato de espaguetis que se debe desenmarañar. Pero en realidad podemos decir que la integración entre los cuatro países ya existe, porque entre sí tienen prácticamente arancel cero" (Carlos Ronderos $\left.{ }^{4}\right)$.

\section{La economía de los miembros y Perú}

En términos demográficos, Chile es el país que tiene menos habitantes de los países miembros de la Alianza y el PBI per cápita -al 2013- con mejores resultados. México es el país que tiene el PBI más alto de los cuatro países integrantes; la suma de los tres restantes es menor de lo que produce México. En lo que respecta al comercio internacional, México es el país que representa los mayores montos, tanto por el lado de las exportaciones como de las importaciones.

3 Carlos Ronderos. La Alianza del Pacífico aún está lejos de lograr integrarse con Asia. Publicado en http:// www.larepublica.pe/23-11-2014/la-alianza-del-pacifico-aun-esta-lejos-de-lograr-integrarse-con-asia. Consultado el 29 de noviembre del 2014.

4 Carlos Ronderos es gerente de desarrollo regional para Latinoamérica de la Asociación The World Trade Centers. Ex ministro de Comercio Exterior de Colombia. Economista con estudios de doctorado en la Universidad de Glasgow, Escocia. 


\section{Pedro Barrientos Felipa}

CUADRO 1

La economía en los países de la Alianza

\begin{tabular}{|l|c|c|c|c|c|}
\hline \multirow{2}{*}{ Países } & \multirow{2}{*}{$\begin{array}{c}\text { Habitantes } \\
\text { (millones) - }\end{array}$} & \multirow{2}{*}{$\begin{array}{c}\text { PBI 2013 } \\
\text { (corriente) }\end{array}$} & \multirow{2}{*}{$\begin{array}{c}\text { PBI } \\
\text { per cápita }\end{array}$} & \multicolumn{2}{|c|}{$\begin{array}{c}\text { Comercio internacional 2013 } \\
\text { US\$ millones }\end{array}$} \\
\cline { 5 - 6 } & $2013(e)$ & US\$ millones & (ppp 2013) & Exportaciones & Importaciones \\
\hline Chile & 17.6 & 281,660 & 19,105 & 77,400 & 74,990 \\
\hline Colombia & 47.2 & 369,225 & 11,087 & 58,800 & 56,620 \\
\hline México & 118.4 & $1,327,000$ & 15,563 & 380,1180 & 381,210 \\
\hline Perú & 30.9 & 206,700 & 11,149 & 41,900 & 41,110 \\
\hline
\end{tabular}

Fuente y elaboración: Alianza del Pacífico (2014)

Ronderos (2014) también expresa que "la agroindustria es nuestra gran oportunidad. Pero también deberíamos apuntar a los servicios. En software ya hay vestigios importantes y en servicios logísticos Chile está muy avanzado. En realidad la industria de servicios se está desagregando y allí la AP tiene una gran oportunidad que debe aprovechar porque genera mucho valor agregado". Hay oportunidades comerciales al formarse la Alianza del Pacífico. Para Perú puede ser el sector de la agricultura, actividad en la cual está ingresando con diversos productos que más tienen tendencia hacia el comercio exterior.

\section{CUADRO 2}

El PBI per cápita de los miembros de la Alianza del Pacífico vs. Brasil

\begin{tabular}{|l|c|c|c|}
\hline Países & $\begin{array}{c}\text { PBI (nominal) per cápita - } \\
\text { Estimado 2012 } \\
\text { (US\$) - Anual (1) }\end{array}$ & $\begin{array}{c}\text { PBI per cápita - Paridad } \\
\text { de poder adquisitivo - } \\
\text { Estimado 2014 (2) }\end{array}$ & $\begin{array}{c}\text { Índice igualdad de ingreso } \\
\text { (Coeficiente de Gini) (3) }\end{array}$ \\
\hline Chile & 15,415 & 20,313 & 0.521 \\
\hline Colombia & 8,127 & 11,284 & 0.559 \\
\hline México & 10,514 & 15,931 & 0.472 \\
\hline Perú & 6,572 & 11,403 & 0.481 \\
\hline Brasil & 12,339 & 12.340 & 0.547 \\
\hline
\end{tabular}

Fuente: (1) International Monetary Fund. Publicado en http://www.imf.org/external/pubs/ft/weo/2012/02. Consultado el 30 de julio del 2012. (2) International Monetary Fund http://www.imf.org/external/pubs/ $\mathrm{ft} /$ weo/2013/02. Consultado el 30 de julio del 2014. (3) Publicado en http://es.wikipedia.org/wiki/ Anexo:Pa\%C3\%ADses_por_igualdad_de_ingreso\#Am.C3.A9rica Consultado el 30 de Julio del 2014 


\section{Pensamiento Crítico Vol. 20. No 2}

Al realizar una lista de los países que son parte de la Alianza del Pacífico y se ordena según el producto bruto interno (PBI) a precios nominales per cápita, que es resultado de la suma de todos los bienes y servicios finales producidos por un país en un año, dividido por la población estimada para mediados del mismo año, las cifras manifiestan (en el cuadro 1) que Chile tiene el mejor resultado a fines del 2012.

Cuando se clasifica a los miembros de la Alianza según el PBI a valores de paridad de poder adquisitivo (PPA), la que se calcula basándose en la suma de todos los bienes y servicios finales producidos por un país en un año, en relación con una canasta de precios estandarizada, ponderada o cotizada dentro del propio mercado interno de los Estados Unidos, país que se toma como referencia en las comparaciones internacionales realizadas según la metodología PPA. Es por eso justamente que el PBI nominal estadounidense es siempre idéntico a su propio PBI PPA (para un año determinado y según una misma fuente). En este caso, Chile tiene el mejor resultado.

\section{Principales acuerdos de integración económica en América Latina}

Los países deben estar seguros, en especial sus dirigentes, que es necesario estar integrados económicamente con otros países. Latinoamérica es la parte del continente que está tomando este desafío y ha logrado resultados que pueden ser mejores. La globalización lleva a tomar esta decisión de enfrentar los desafíos de manera conjunta a otros países. Sin embargo, en el análisis debe considerarse que no todos los países que están o son parte del proceso de integración tienen las mismas condiciones.

\section{Asociación Latinoamericana de Integración - ALADI}

Es el mayor grupo ${ }^{5}$ latinoamericano de integración. Sus trece países miembros comprenden a Argentina, Bolivia, Brasil, Chile, Colombia, Cuba, Ecuador, México, Panamá, Paraguay, Perú, Uruguay y Venezuela, que representa en conjunto 20 millones de kilómetros cuadrados y más de 510 millones de habitantes. La ALADI propicia la creación de un área de preferencias económicas en la región, con el objetivo final de lograr un mercado común latinoamericano, mediante tres mecanismos:

5 Fuente: Publicado en http://www.aladi.org/ 


\section{Pedro Barrientos Felipa}

- Una preferencia arancelaria regional que se aplica a productos originarios de los países miembros frente a los aranceles vigentes para terceros países.

- Acuerdos de alcance regional (comunes a la totalidad de los países miembros).

- Acuerdos de alcance parcial, con la participación de dos o más países del área.

Tanto los acuerdos regionales como los de alcance parcial pueden abarcar materias diversas como desgravación arancelaria y promoción del comercio; complementación económica; comercio agropecuario; cooperación financiera, tributaria, aduanera, sanitaria; preservación del medio ambiente; cooperación científica y tecnológica; promoción del turismo; normas técnicas; y muchos otros campos previstos a título expreso o no en el TM 80.

\section{Mercado Común del Sur - MERCOSUR}

El Mercado Común del Sur -MERCOSUR- está integrado por la República Argentina, la República Federativa de Brasil, la República del Paraguay, la República Oriental del Uruguay, la República Bolivariana de Venezuela y el Estado Plurinacional de Bolivia. El MERCOSUR tiene como Estados asociados a Chile, Colombia, Perú, Ecuador, Guyana y Surinam. La participación de los Estados asociados en las reuniones del MERCOSUR y la suscripción de Acuerdos se rigen por lo establecido en las Decisiones $N^{\circ} 18 / 04,28 / 04$ y 11/13.

Peña (2011, p. 110) expresa que, de acuerdo a la experiencia adquirida, la que está influenciada por los cambios que se han llevado a cabo, es una oportunidad para reflexionar sobre el futuro de Mercosur. En tal sentido, propone las siguientes consideraciones: 1) tomar en cuenta los cambios profundos de circunstancias y necesidades producidos desde que se firmó el Tratado de Asunción; 2) hacer una revisión de la validez de la idea estratégica que impulsa la creación del Mercosur; y 3) la metodología del trabajo conjunto entre los países socios, tomando en cuenta que mucho se ha aprendido en los años pasados sobre cómo cooperar en distintos planos. Estos tres aspectos son relevantes para el futuro de Mercosur. 


\section{Pensamiento Crítico Vol. 20. No 2}

\section{Comunidad Andina de Naciones - CAN}

Se propone como una comunidad ${ }^{6}$ de países que se une voluntariamente con el objetivo de alcanzar un desarrollo integral, más equilibrado y autónomo, mediante la integración andina, sudamericana y latinoamericana. Los países que la integran son: Bolivia, Colombia, Ecuador y Perú, quienes están unidos por el mismo pasado, una variada geografía, una gran diversidad cultural y natural, así como por objetivos y metas comunes. Los cuatro países presentan una gran diversidad cultural, étnica y lingüística. Poseemos riquísimas manifestaciones folklóricas y artísticas que nos unen aún más.

La Comunidad Andina está conformada por órganos e instituciones que están articulados en el Sistema Andino de Integración, más conocido como el SAI. Este sistema hace que la CAN funcione casi como lo hace un Estado. Es decir, cada una de estas instancias tiene su rol y cumple funciones específicas, por ejemplo: el Consejo Presidencial Andino, conformado por los presidentes de Bolivia, Colombia, Ecuador y Perú, está a cargo de la dirección política de la CAN; el Consejo Andino de Ministros de Relaciones Exteriores formula la política exterior de los países andinos en asuntos relacionados con la integración y, de ser necesario, coordinan posiciones conjuntas en foros o negociaciones internacionales; la Comisión, conformada por delegados plenipotenciarios o con plenos poderes, se encarga de formular, ejecutar y evaluar la política de integración en temas de comercio e inversiones y genera normas que son de obligatorio cumplimiento para los cuatro países.

\section{Asia - Pacific Economic Cooperation - APEC}

APEC (Asia-Pacific Economic Cooperation, en español Foro de Cooperación Económica Asia-Pacífico) es un foro multilateral creado en 1989, con el fin de consolidar el crecimiento y la prosperidad de los países del Pacífico, que trata temas relacionados con el intercambio comercial, coordinación económica y cooperación entre sus integrantes. Como mecanismo de cooperación y concertación económica, está orientado a la promoción y facilitación del comercio, las inversiones, la cooperación económica y técnica y al desarrollo económico regional de los países y territorios de la cuenca del océano Pacífico.

6 Fuente consultada: http://www.comunidadandina.org/Seccion.aspx?id=189\&tipo=QU\&title=somoscomunidad-andina 


\section{Pedro Barrientos Felipa}

APEC es el foro económico que tiene como principal objetivo apoyar el crecimiento económico sostenible y la prosperidad en la región Asia-Pacífico. Busca construir una comunidad dinámica y armoniosa defendiendo el libre comercio y la inversión, promover y acelerar la integración económica regional, a la vez que fomenta la cooperación económica y técnica, así como la mejora de la seguridad humana y facilitar un ambiente de negocios favorable y sostenible. La suma del producto nacional bruto de las 21 economías que conforman APEC equivale al $56 \%$ de la producción mundial, en tanto que en su conjunto representan el $46 \%$ del comercio global.

\section{Unión de Naciones Sudamericanas - UNASUR}

Es un organismo internacional, conformado por los doce países de la región sudamericana: Argentina, Bolivia, Brasil, Colombia, Chile, Ecuador, Guyana, Paraguay, Perú, Suriname, Uruguay y Venezuela. Tiene por objetivo construir un espacio de integración en lo cultural, económico, social y político, respetando la realidad de cada nación. Su desafío es eliminar la desigualdad socioeconómica, alcanzar la inclusión social, aumentar la participación ciudadana, fortalecer la democracia y reducir las asimetrías existentes, considerando la soberanía e independencia de los Estados.

\footnotetext{
"Mucho se ha dicho respecto al sesgo o rasgo político de la Alianza del Pacífico. Pues la verdad es que no lo tiene. Fue ideada como mecanismo para la profundización de la integración económica y comercial a diferencia del ALBA, que es un proceso ideologizado y que, además, realiza poco en materia de integración económica y comercial; difícil, pues, hallar compatibilidades. En cambio, con la Unasur, que es un esquema de integración física y de cooperación política, es posible ser complementarios y trabajar, con razonables expectativas, en el desarrollo de la infraestructura física, y en el diálogo y la coordinación política" (García Belaunde)
}

Ramírez (2013, p. 3) se plantea la pregunta siguiente: ¿tienen estos acuerdos de integración objetivos divergentes? La UNASUR se diseña como uno de los proyectos de integración regional más ambiciosos en Sudamérica. Algunos de los objetivos específicos más importantes que se ha planteado son los siguientes: el fortalecimiento del diálogo político, el desarrollo social y humano, la erradicación del analfabetismo, la integración energética, la construcción de infraestructura para la integración de la región y la integración financiera. Por otro lado, la Alianza del Pacífico tiene como objetivo 


\section{Pensamiento Crítico Vol. 20. No 2}

principal la construcción de un área de integración a través de la libre circulación de bienes, servicios, capitales y personas. En este sentido, los países miembros coinciden en una visión de integración entendida puntualmente como la apertura al comercio, fomento de las inversiones y desarrollo de la libre empresa.

\section{Perspectivas de la Alianza del Pacífico}

La Alianza del Pacífico no es la primera iniciativa que plantea la integración en Latinoamérica, sino que es una adicional dentro de un proceso de actos que buscaron la unidad económica de los países de esta región. Los países integrantes tienen una diversidad de acuerdos bilaterales con otros países de diversas partes del mundo, en los cuales, en ocasiones, también son miembros comunes. Tienen el objetivo común de conformar un área de integración que permita avanzar hacia una etapa que permita la libre circulación de los bienes, servicios, personas y capitales. Lo político es un tema pendiente.

Del Valle (2013, p. 26) manifiesta que este proceso es multidimensional y que los acuerdos a los que se llegarán serán amplios y que es una base para una mayor integración de las economías nacionales, un fortalecimiento del mercado de capitales y ser atractivos para la inversión extranjera directa. Cita además que los factores que contribuyen a la participación de los países miembros en bloques de cadenas productivas y sobre los cuales se debe prestar atención son:

a. Facilitación del comercio y de las inversiones recíprocas: mejoras en la infraestructura, simplificación de los procesos aduaneros.

b. Acceso a los mercados: política arancelaria, regímenes de origen.

c. Armonización normativa en el campo sanitario y fitosanitario, así como en las normas técnicas.

d. Marcos regulatorios para el desarrollo industrial y el establecimiento de cadenas intrarregionales.

e. Inserción internacional basándose en el fomento de la competitividad exportadora: alianzas estratégicas, programas públicos de apoyo empresarial, promoción conjunta. 


\section{Pedro Barrientos Felipa}

Con base en los indicadores económicos de América Latina, se podría señalar que la Alianza del Pacífico constituye la agrupación comercial de mayor tamaño de esta región, con tan solo algo más de un tercio de la población. Para el año 2012 la CEPAL calcula que los 33 países de América Latina y el Caribe sumaban una población de 597 millones de habitantes, de los cuales 210 millones, equivalentes a un 35\%, pertenecerían a la Alianza del Pacífico actual, la cual se ampliaría al $37 \%$ con los dos nuevos Estados parte (218 millones de personas).

El sector empresarial es un protagonista importante en el proceso de integración y los gobiernos deben ser agentes dinámicos para promover su participación y colaboración en el proceso y de esta manera aprovechar las oportunidades que representa este esfuerzo; debe buscar la optimización de los recursos actuales y potenciales. En tal sentido, la cooperación entre los gobiernos debe buscar una trato preferencial en la búsqueda de una coherencia regulatoria, basándose en el respeto mutuo de las políticas locales y los propios objetivos nacionales, lo que no es tarea sencilla pero sí obligatoria para que se desarrollen las cadenas que son útiles para avanzar.

La Alianza del Pacífico incrementa el número de bloques que se han formado para aprovechar tal situación; el proceso de integración debe dar como resultado un proceso sinergético, en donde la suma de las partes (países) tiene mejor resultado si cada uno actúa individualmente. La Alianza tiene sus ventajas sobre los otros bloques económicos latinoamericanos. Se puede asumir, bajo esta última consideración, que apunta a ser el más resaltante bloque económico y comercial de Latinoamérica. Las exportaciones combinadas de los miembros de la Alianza del Pacífico son más grandes

que las del bloque de países sudamericanos del MERCOSUR, liderado por Brasil y Argentina. Pero, internacionalmente, la Alianza del Pacífico viene a constituir un reforzamiento a la capacidad de negociación y de liderazgo que se reconoce en Brasil.

\section{Comentarios finales}

La integración es una condición primaria para aumentar los negocios internacionales de todo nivel. Implica, para los países que son parte de un proceso similar, desafíos para buscar oportunidades y aprovecharlas. En América Latina hay una diversidad de acuerdos económicos entre países, lo que incluye a la Alianza del Pacífico, que origina otro desafío: cómo disminuir el valor relativo de los commodities, así como impulsar la producción de productos con un mayor agregado. 


\section{Pensamiento Crítico Vol. 20. No 2}

Los países que forman la Alianza del Pacífico (Chile, Colombia, México y Perú) son países o mercados emergentes que tienen intereses comunes, crecimientos y políticas internas distintas y también sus propias ventajas respecto a los demás integrantes. Su desventaja comercial internacional es la capacidad de exportación de productos primarios y lo que pueda suceder a estos productos los afecta significativamente. Los países integrantes deben colaborar entre ellos para dar un impulso a productos que les permita tener una mayor independencia comercial y beneficie a sus agentes locales. La agroindustria es una buena oportunidad, así como el turismo.

La Alianza del Pacífico, al privilegiar la integración como instrumento para mejorar el desarrollo económico y social, promover las capacidades de emprendimiento y conformar un espacio ampliado y competitivo que sea atractivo a las inversiones y el comercio, es conveniente que promueva nuevas cadenas productivas que hagan que el bloque sea atractivo para el mundo y generen mayores oportunidades de encadenamientos, de inversión y de incorporación más intensa a las corrientes globales de comercio de bienes y servicios, para que ofrezcan nuevas oportunidades de negocios y empleo a sus economías.

\section{Referencias bibliográficas}

Acuña Bolívar, Juan (2013). La Alianza del Pacífico en la Integración Latinoamericana y Caribeña. Sistema Económico y del Caribe - SELA.

Barrientos Felipa, Pedro (2009). El marketing en el Perú y la globalización. Semestre económico, Universidad de Medellín. Volumen 12, № 23, pp. 59 - 75.

Burgos, Yonny; Coasaca, Juan; Valcarcel, Violeta. (2003). La globalización: análisis e impacto en el Perú. Lima: Revista Producción y Gestión, Facultad de Ingeniería Industrial - UNMSM. pp. 20 - 26.

Del Valle Márquez, Julybeth (2013). Perspectivas de la Alianza del Pacífico para la generación de encadenamientos productivos regionales. Universidad de Chile, Instituto de Estudios Internacionales. Santiago de Chile. 


\section{Pedro Barrientos Felipa}

Friedman, Thomas (2006). La tierra es plana: breve historia del mundo globalizado del siglo XXI. Ediciones Martínez Roca S. A. Madrid.

Jaramillo, Felipe; Silva-Jáuregui, Carlos (2011). Perú en el umbral de una nueva era, lecciones y desafíos para consolidar el crecimiento económico y un desarrollo más incluyente. Banco Mundial.

Ohmae, Kenichi (2008). El nuevo escenario global, desafíos y oportunidades en un mundo sin fronteras. McGraw-Hill Interamericana de España S. A. U.

Peña, Félix (2011). El MERCOSUR y las complejidades de la integración regional. El Mercosur, veinte años después y su futuro. Pp. 105-120. Editorial Teseo. Buenos Aires.

Ramírez, Javier (2013). Bibliografía anotada sobre la Unión Sudamericana de Naciones y la Alianza del Pacífico. Pontifica Universidad Católica del Perú - Escuela de Gobierno y Políticas Públicas. Lima.

Rodrik, Dani (2011). La paradoja de la globalización: democracia y el futuro de la economía mundial. Antoni Bosch Editor. Barcelona.

San Francisco, Alejandro (2013). La Alianza del Pacífico: un compromiso de futuro. Fundación Ciudadanía y Valores. Madrid.

Serrano Monteavaro, Miguel (2012). La Alianza del Pacífico. Instituto Español de Estudios Estratégicos. Documento Informativo 46/2012. Madrid.

Sistema Económico Latinoamericano y del Caribe - SELA (2013). La Alianza del Pacífico en la Integración Latinoamericana Caribeña. SELA. Caracas.

Vásquez Barquero, Antonio (2005). Las nuevas fuerzas del desarrollo. Antoni Bosch Editor S. A. Barcelona. 